\title{
LA COLABORACIÓN EN LA RED: HACIA UNA DEFINICIÓN DE APRENDIZAJE COLABORATIVO EN ENTORNOS VIRTUALES
}

Resumen: En este artículo se presenta la Red temática sobre aprendizaje colaborativo en entornos virtuales (RACEV), en la que trabajan conjuntamente un grupo de investigadores pertenecientes a diferentes instituciones universitarias españolas. Para ello, se describen las principales acciones llevadas a cabo en el marco de dicha red para focalizar en aspectos de carácter teórico sobre esta metodología que conducen a una definición sobre el aprendizaje colaborativo en entornos virtuales.

Palabras clave: red temática; aprendizaje en línea; aprendizaje colaborativo; aprendizaje cooperativo; aprendizaje colaborativo en entornos virtuales.

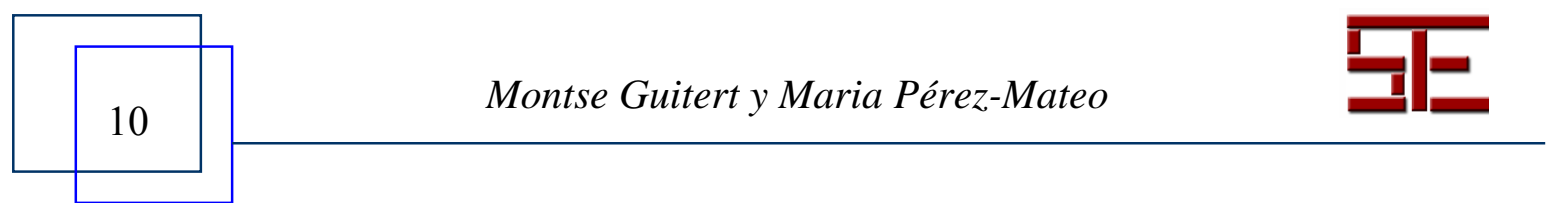




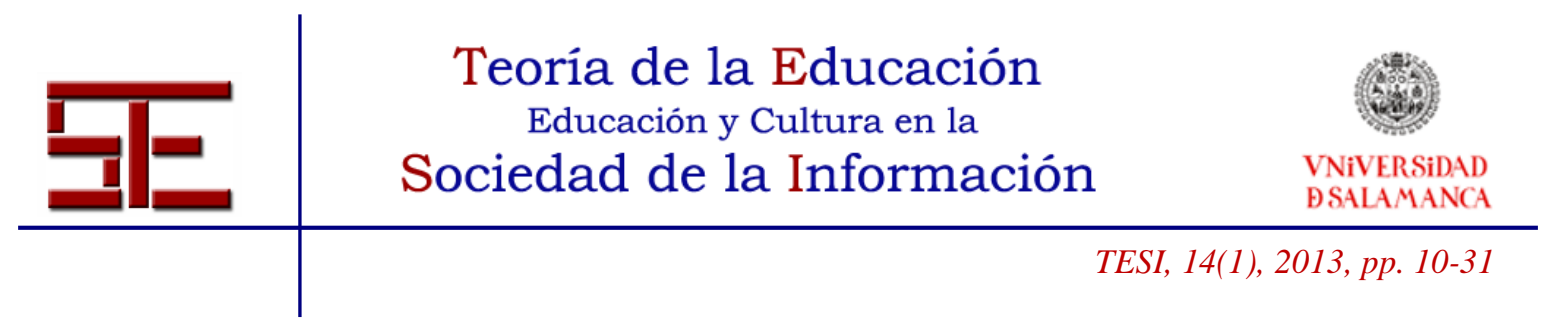

\title{
NETWORKED COLLABORATION: TOWARDS A DEFINITION OF ONLINE COLLABORATIVE LEARNING
}

\begin{abstract}
This article presents the thematic network on collaborative learning in virtual environments (RACEV), in which a group of researchers from different Spanish universities work together. It describes the main activities carried out within the framework of the network focusing on the theoretical aspects of this methodology. This action leads to a definition of collaborative learning in virtual environments.
\end{abstract}

Key words: thematic network; online learning; collaborative learning; cooperative learning; online collaborative learning.

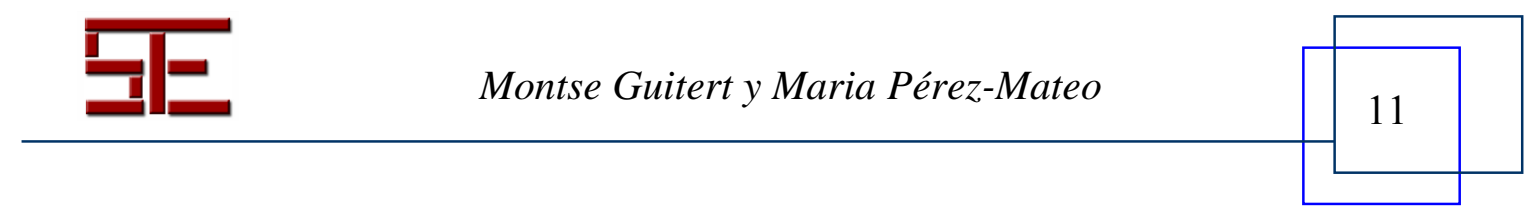




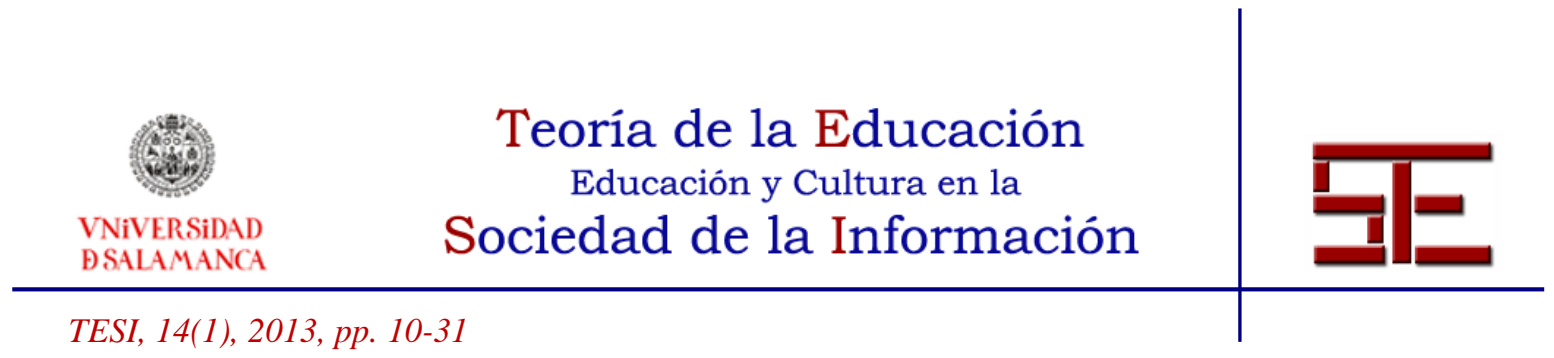

\section{LA COLABORACIÓN EN LA RED: HACIA UNA DEFINICIÓN DE APRENDIZAJE COLABORATIVO EN ENTORNOS VIRTUALES}

Fecha de recepción: 23/12/2012; fecha de aceptación: 01/02/2013; fecha de publicación: 28/02/2013

Montse Guitert

mguitert@uoc.edu

Universitat Oberta de Catalunya

María Pérez-Mateo

mperez-mateo@uoc.edu

Universitat Oberta de Catalunya

\section{1.- INTRODUCCIÓN}

Los procesos de colaboración en el ámbito educativo no son nuevos. Lara (2001) señala que los primeros estudios realizados sobre la necesidad de desarrollar este tipo de procesos en el aprendizaje datan de los años cuarenta. No obstante, desde entonces su desarrollo no ha sido lineal sino que ha experimentado diferentes etapas en función del interés depositado en éste $u$ otros tipos de aprendizaje como el competitivo o el individualista. Es a partir de los años 70 que el aprendizaje colaborativo vuelve a cobrar interés (Lara, 2001).

En el marco de la sociedad red la colaboración ha dado otro giro, teniendo en cuenta que se articula ajustadamente con la estructura en que se basa Internet: la red (Suárez, 2009). La colaboración incluso se contempla como una característica clave del aprendizaje en línea (Badia y García, 2006; Garrison, 2006; Gunawardena y Zittle, 1997; Harasim et al., 2000; Kirschner, 2002; Majó y Marquès, 2002). En ese sentido, Dillenbourg (2003) señala que el aprendizaje colaborativo mediado por ordenador ha ganado adeptos en los últimos años hasta el punto de que algunos aseguran que es el uso dominante de la tecnología en educación. La colaboración incluso aparece al tratar de conceptualizar los procesos de enseñanza-aprendizaje en el entorno virtual. En esta línea, McConnell (2006) hace referencia al "e-learning colaborativo en red". También el EEES reconoce la importancia de estos procesos para desenvolverse en la sociedad actual, definiendo el trabajo en equipo como una competencia genérica dentro del proyecto Tuning Educational Structures in Europe (González y Wagenaar, 2003).

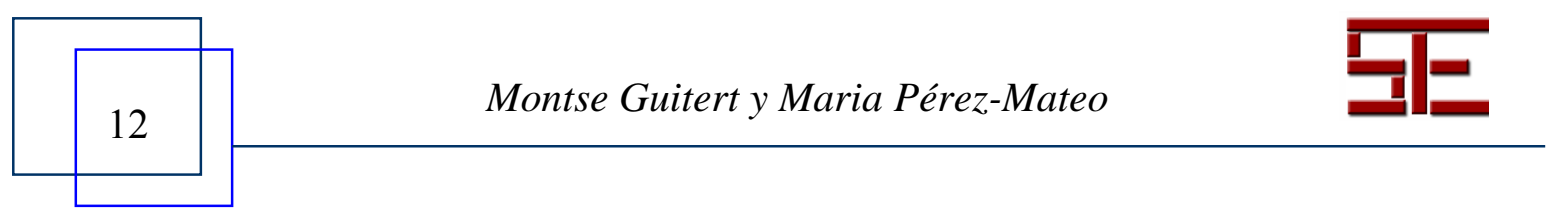




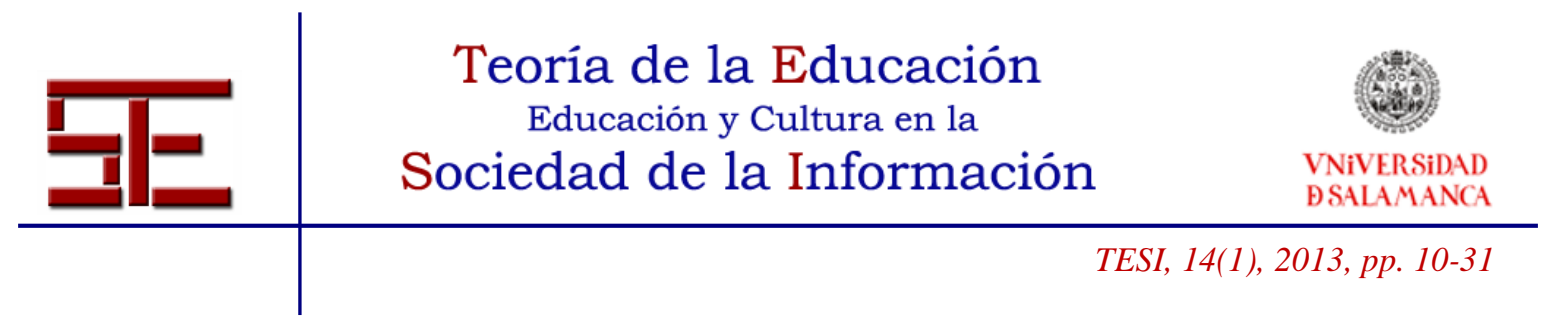

Los beneficios del aprendizaje colaborativo están ampliamente documentados en la literatura del ámbito. Roberts (2005) agrupa los principales efectos que el aprendizaje colaborativo puede aportar a los estudiantes de la siguiente manera:

- Académicos, puesto que enfatiza en la participación y construcción activa de conocimiento, promoviendo habilidades de alto orden de pensamiento e incrementando los resultados de la clase;

- Sociales, teniendo en cuenta que fomenta la creación de una atmósfera positiva para el aprendizaje a la vez que permite desarrollar un sistema de apoyo social para los estudiantes, fomentando la comprensión y enseñanza entre ellos;

- Psicológicos, ya que desarrolla actitudes positivas hacia los profesores y puede incrementar la autoestima de los estudiantes.

Atendiendo a la categorización propuesta por Roberts (2005) y a partir del análisis de la literatura en el ámbito ${ }^{1}$, la Figura 1 identifica los principales beneficios asociados al aprendizaje colaborativo.

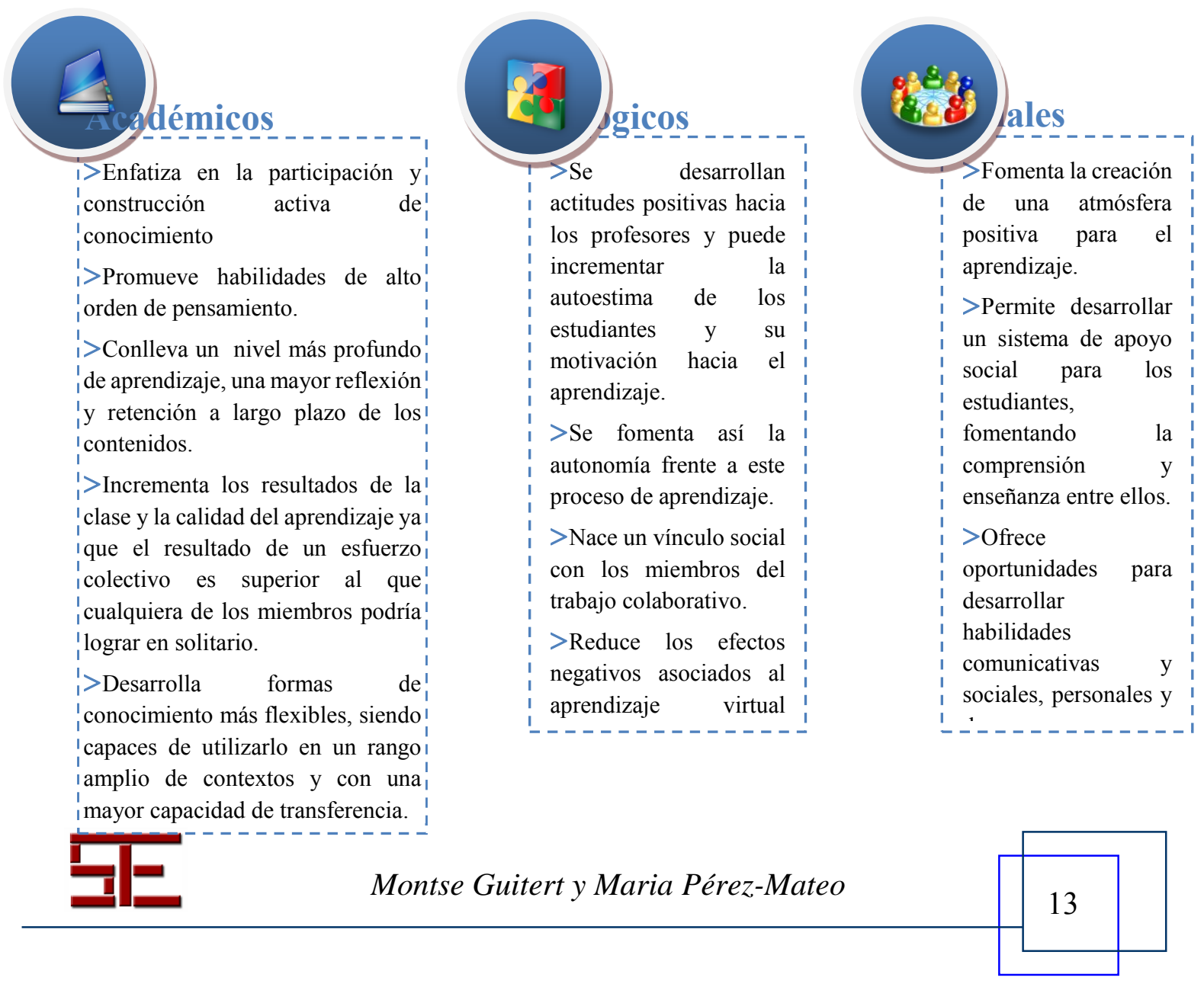




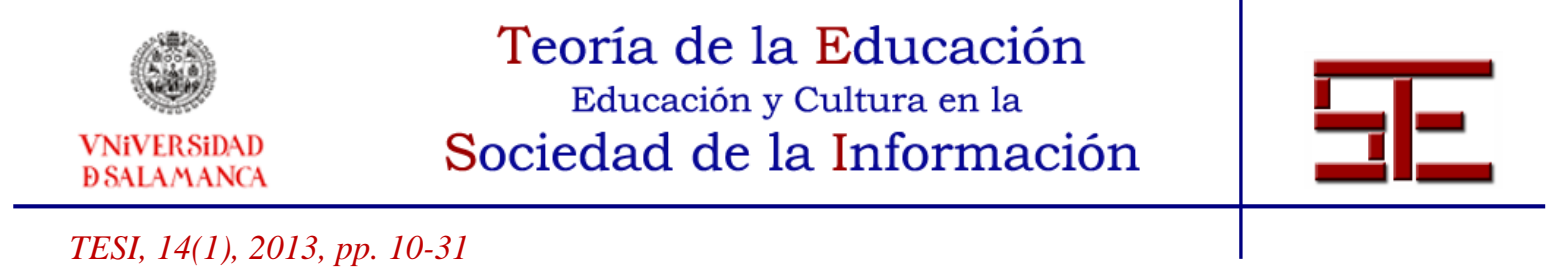

Figura 1. ¿Para qué colaborar? Beneficios del aprendizaje colaborativo

Al mismo tiempo, numerosas investigaciones remarcan que situar a los estudiantes en grupos no garantiza la colaboración (Brush, 1998; Dillenbourg, 2002; Johnson y Johnson, 1989). Esto conlleva la necesidad de reparar en su planteamiento y el soporte ofrecido al estudiante.

Es en este contexto conceptual y en la interacción de un grupo de docentes e investigadores universitarios con un amplio bagaje en este ámbito de estudio donde surge la Red temática sobre aprendizaje colaborativo en entornos virtuales (RACEV).

\section{2.- RED TEMÁTICA SOBRE APRENDIZAJE COLABORATIVO EN ENTORNOS VIRTUALES (RACEV)}

Compartir un interés en torno al aprendizaje colaborativo en red llevó a un conjunto de investigadores pertenecientes a diferentes universidades españolas a configurar la Red temática sobre aprendizaje colaborativo en entornos virtuales.

Como red temática, la RACEV se circunscribe en la convocatoria de Acciones Complementarias del Ministerio de Educación y Ciencia, Acciones Complementarias (Ref. EDU2010-09535-E)². Su duración es de 3 años (2011-2013), por lo que se encuentra actualmente iniciando su último período.

\section{1.- Objetivos de RACEV}

RACEV tiene por objetivo facilitar el aprendizaje colaborativo en línea entre estudiantes a través del análisis de los fenómenos de interacción social (colaborativa o cooperativa) a fin de proporcionar estrategias pedagógicas a los docentes.

La red focaliza, por tanto, en los procesos de aprendizaje más allá del contexto en el cual se desarrolla o las herramientas tecnológicas empleadas.

Partiendo de este objetivo general, RACEV se plantea:

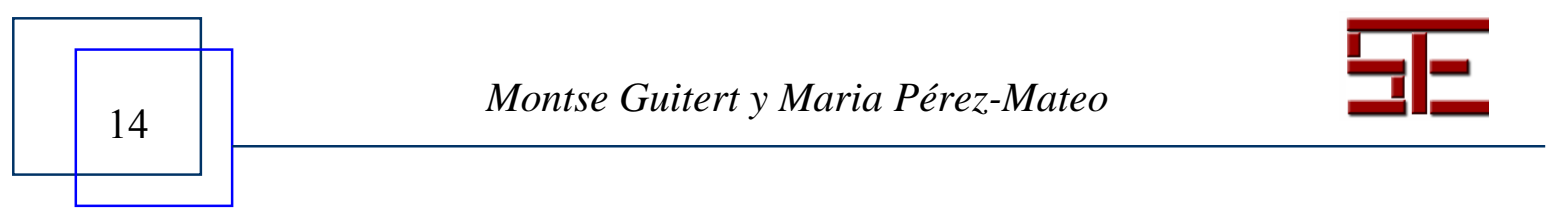




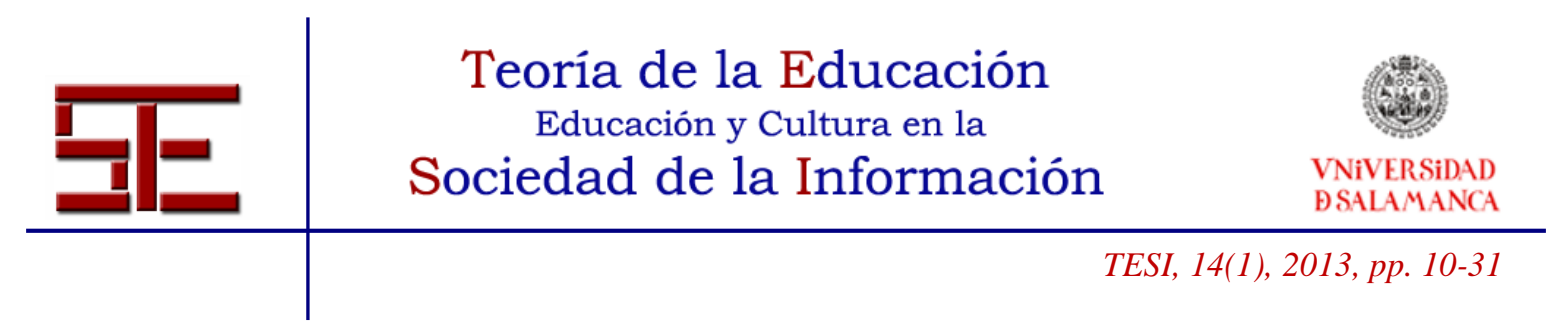

- Consolidar una comunidad de investigación en torno al aprendizaje colaborativo a nivel estatal.

- Crear y mantener un portal orientado al aprendizaje colaborativo desde un punto de vista pedagógico.

- Desarrollar una línea de investigación en torno al aprendizaje colaborativo en red.

- Difundir los resultados obtenidos fruto de la actividad de la red temática más allá del portal virtual.

Su finalidad última es mejorar los procesos de aprendizaje colaborativo que tienen lugar en la práctica educativa.

\section{2.- Participantes de RACEV}

El equipo de RACEV está formado por investigadores con un amplio bagaje en el ámbito del aprendizaje en entornos virtuales. Su principal potencial reside en el enfoque pedagógico, el cual constituye el punto de partida de la red temática.

Quince investigadores doctores pertenecientes a nueve instituciones españolas forman parte de esta red:

- Montse Guitert (investigadora principal de RACEV), María Pérez-Mateo, Marc Romero, Teresa Romeu y Sandra Ramírez de la Universidad Abierta de Catalunya $(\mathrm{UOC})^{3}$. Como institución que lleva a cabo los procesos educativos de manera íntegramente virtual, la UOC dispone de una amplia experiencia en el ámbito de la colaboración en red.

- Begoña Gros e Iolanda García de la Universidad de Barcelona (UB).

- Joan Domingo de la Universidad Politécnica de Catalunya (UPC).

- Joan Rué de la Universidad Autónoma de Barcelona (UAB).

- Jesús Salinas de la Universidad de las Islas Baleares (UIB).

- Manuel Area y Amador Guarro de la Universidad de la Laguna (ULL).

- Bartolomé Rubia de la Universidad de Valladolid (UVA).

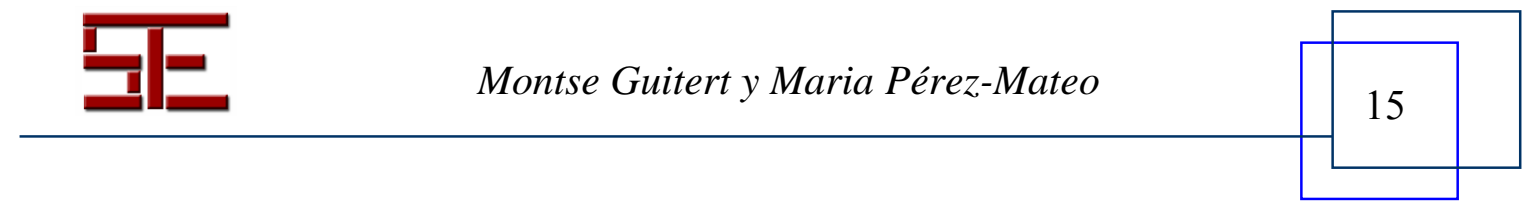




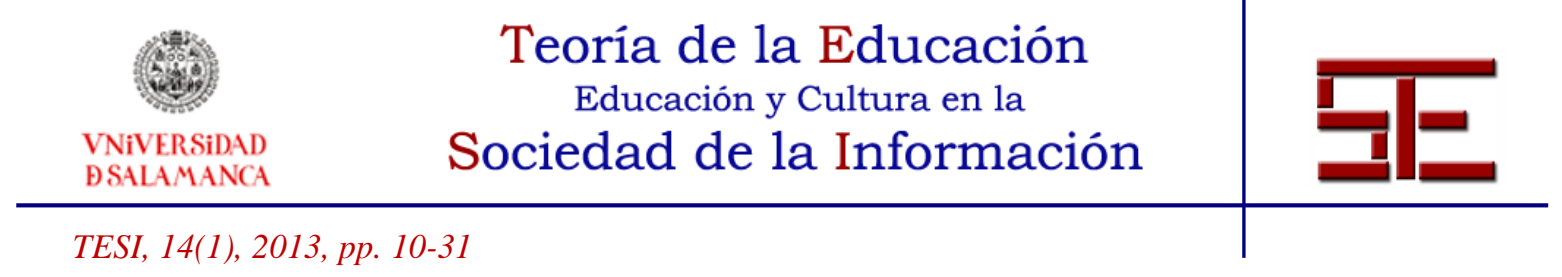

- Cristóbal Suárez de la Universidad de Valencia (UV).

- Pedro Román de la Universidad de Sevilla (US).

Las actividades de la red se desarrollan a través del entorno virtual. Los encuentros presenciales se reducen a una jornada anual.

\section{3.- Fases de RACEV}

En el procedimiento de desarrollo de RACEV se identifican tres fases concretas. Su sucesión intercala las contribuciones teóricas con las prácticas o aplicadas (Figura 2).

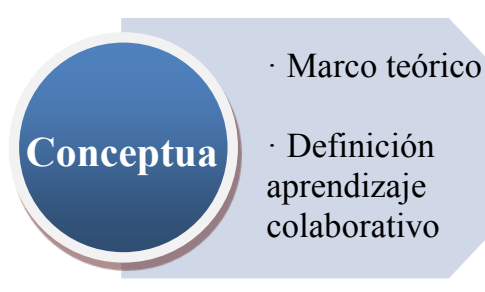

1

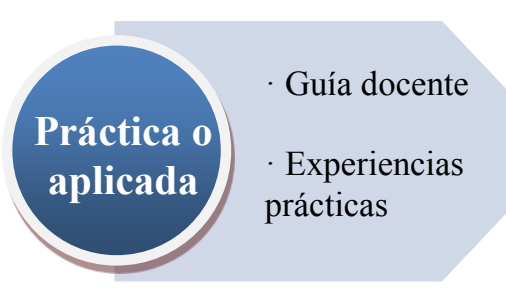

2

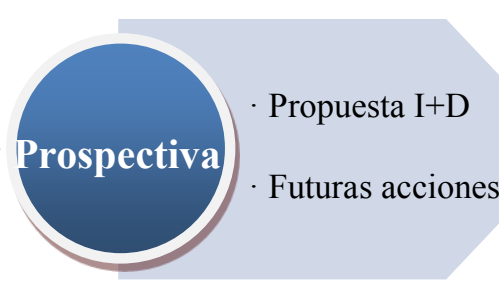

3

Figura 2. Procedimiento de desarrollo de las acciones de RACEV

Una primera fase de carácter conceptual, orientada a comprender en profundidad el aprendizaje colaborativo en entornos virtuales a partir de la elaboración de un marco teórico. Este proceso nos condujo a una definición consensuada de aprendizaje colaborativo.

Partiendo de este marco teórico, la segunda fase se centra en aplicar el aprendizaje colaborativo desde una doble vertiente.

Por un lado, desde la práctica o experiencia educativa. En esta línea, se compartieron diferentes propuestas de aprendizaje colaborativo en diferentes ámbitos y diferentes condiciones, las cuales incluyen tanto la descripción del planteamiento pedagógico como

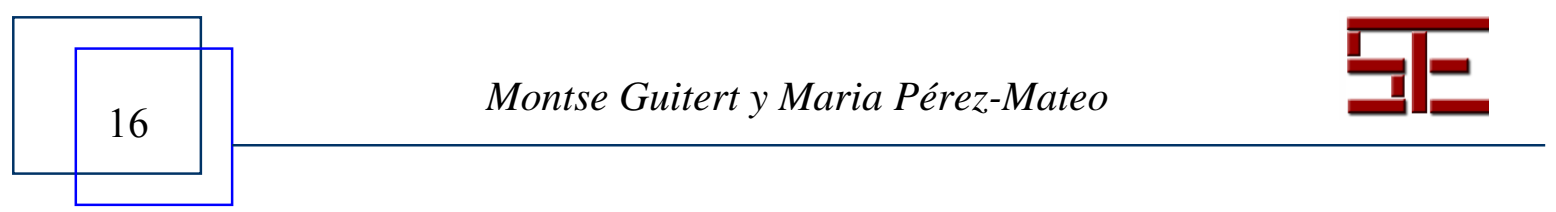




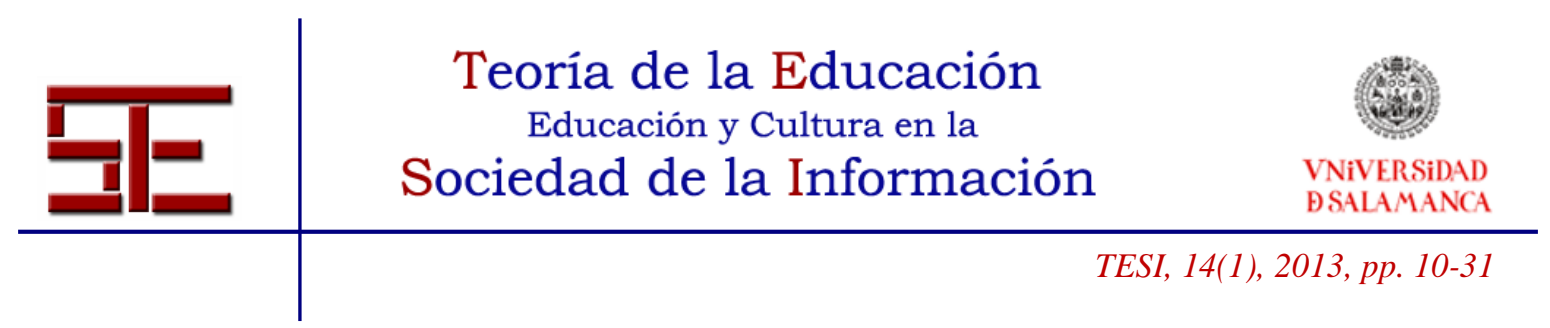

un análisis sobre las mismas, orientado a analizar el grado de colaboración, el impacto en términos de aprendizaje y los principales puntos fuertes y débiles detectados.

Por otro lado, desde el punto de vista docente, del diseño del aprendizaje colaborativo y las estrategias para desarrollarlo. La Guía sobre aprendizaje colaborativo en entornos virtuales es el resultado de este proceso. Esta guía pretende reunir los factores que ayudan a desarrollar el aprendizaje colaborativo en educación superior a la vez que facilitar pautas para su diseño a fin de extraer el máximo provecho de los procesos colaborativos. De esta manera, facilita herramientas, conceptos y pautas a docentes y profesionales de la educación que lleven a cabo actividades y trabajos colaborativos en entornos virtuales o blended (semi-presenciales) en el ámbito de la Educación Superior. También incluye una propuesta de ficha modelo con los elementos a definir y planificar para diseñar una actividad colaborativa.

La tercera fase consiste en plantear un plan estratégico para continuar las acciones como red más allá del reconocimiento oficial por parte del Ministerio de Ciencia e Innovación. En esta línea, se está trabajando actualmente en una propuesta de proyecto competitivo a nivel nacional orientado al aprendizaje colaborativo en entornos virtuales. También se definirán líneas futuras de trabajo conjunto.

En este artículo nos centramos en la primera fase de la RACEV, de carácter conceptual.

La primera acción como red se orientó a realizar una revisión bibliográfica a fin de consensuar entre los participantes el concepto de aprendizaje colaborativo a la vez que poner a disposición de los docentes y personas interesadas en educación un marco teórico de referencia que fundamente las prácticas colaborativas.

El marco teórico incluye una definición de aprendizaje colaborativo en entornos virtuales; una referencia a los términos cooperativo y colaborativo; principales perspectivas teóricas que sustentan tales prácticas; elementos clave para comprender el aprendizaje colaborativo virtual; principales beneficios asociados a esta metodología de aprendizaje; las etapas que atraviesa un grupo virtual; herramientas para desarrollar este tipo de procesos; y, por último, el aprendizaje colaborativo virtual desde la perspectiva del docente.

Este proceso evidenció asimismo dos aspectos controvertidos o sobre los que no existe un consenso generalizado.

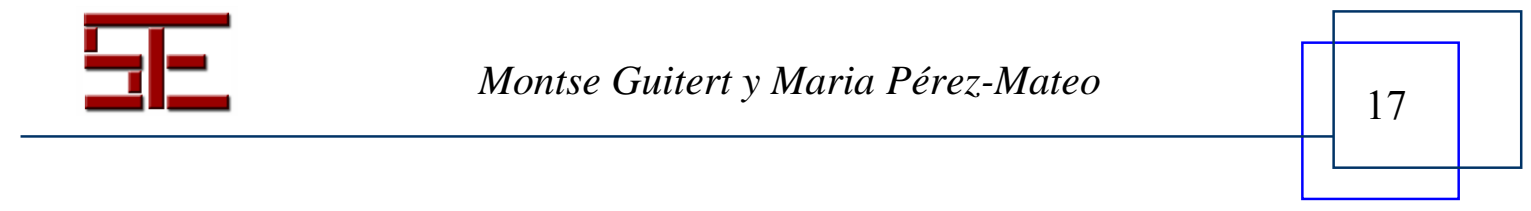




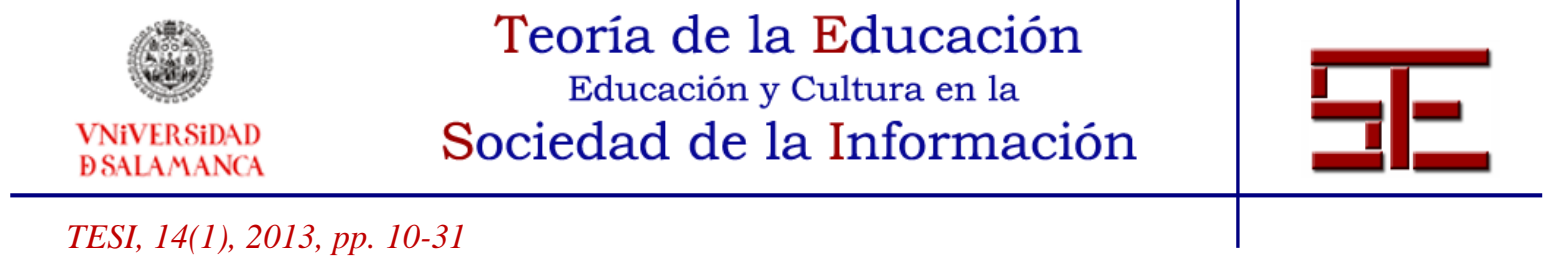

Por un lado, sobre dos términos frecuentemente empleados en la literatura del ámbito: cooperar y colaborar.

Y, por otro lado, se observó que los investigadores tienden a elaborar su propia definición de aprendizaje colaborativo.

Atendiendo a ambos elementos, en este artículo nos centramos en aportar argumentos que ayuden a esclarecer la disyuntiva entre los términos cooperar y colaborar a la vez que aportar una definición consensuada por los miembros RACEV sobre el concepto de aprendizaje colaborativo en entornos virtuales.

\section{3.- ¿COOPERAR O COLABORAR?}

Es frecuente en la literatura educativa la referencia a los términos cooperativo o colaborativo. Pero, ¿se trata del mismo concepto? ¿En qué se asemejan o difieren?

El análisis de dicha literatura evidencia que algunas veces se utilizan como términos diferenciados y otras como sinónimos.

Cabe destacar que las diferencias y similitudes entre ambos términos son todavía hoy causa de debate entre los expertos del ámbito. De hecho, como apuntan Kreijns et al. (2003), la cuestión de qué es aprendizaje colaborativo y cooperativo, así como cuáles son sus diferencias y similitudes, parece ser casi una discusión irresoluble.

Si bien no es pretensión de RACEV dar respuesta a tales disyuntivas, abordamos esta cuestión a fin de argumentar nuestra posición en este aspecto para justificar qué concepto adoptaremos y con qué significado.

\section{1.- Diferencias entre cooperar y colaborar}

En la literatura del ámbito encontramos una cantidad importante de estudios que analizan las diferencias entre los términos "cooperar" y "colaborar", afirmando que ambos enfoques presentan algunas características que los diferencian notoriamente (Dalsgaard y Paulsen, 2009; Holliman y Scanlon, 2006; Panitz, 1997; Thompson, 2005; Zañartu, 2003; Crook, 1998).

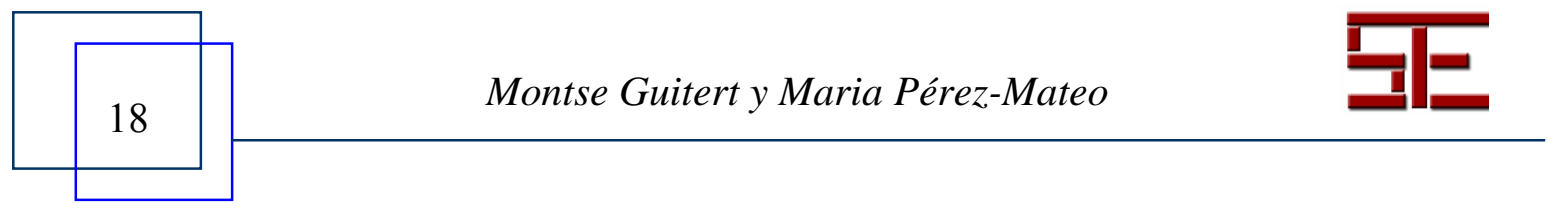




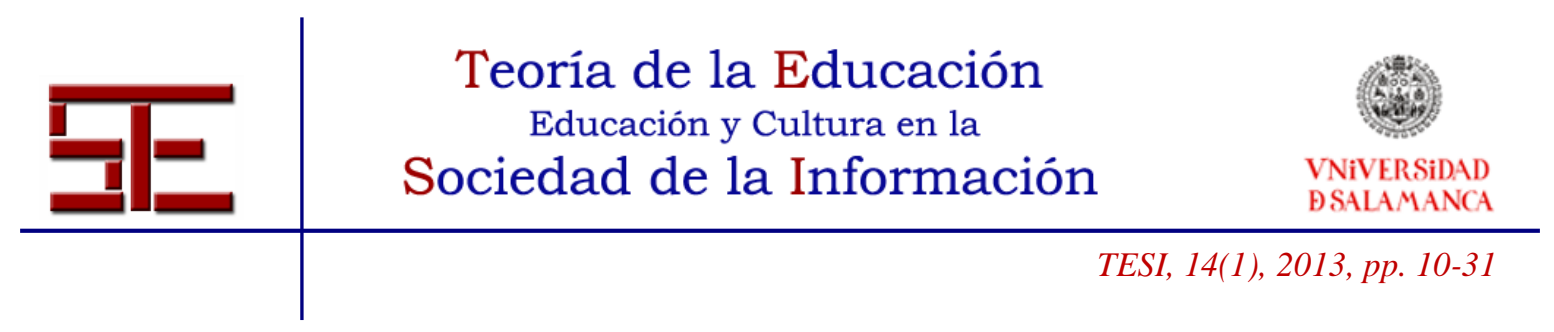

De acuerdo con dichos estudios, se trata de modelos diferentes que surgen a partir de tradiciones educativas distintas. John Myers (1991, citado por Ovejero, 1990) apunta que en la diferenciación entre estos dos conceptos no podemos dejar de lado la influencia de las raíces etimológicas y semánticas. Según este autor, la cooperación tendría sus raíces en el continente americano y, más concretamente, en EE.UU; por el contrario, la colaboración nace en el continente europeo, siendo especialmente desarrollada por la tradición anglosajona.

\section{2.- Origen y concepto de cooperación}

El aprendizaje cooperativo tiene sus raíces en la tradición educativa americana y, más concretamente, en la estadounidense. Como término y concepto, la cooperación proviene de una visión pedagógica basada en el aprendizaje entre iguales o peer to peer.

Jean Piaget destaca como el precursor de este modelo. Efectivamente, Piaget (1923, 1932, 1947, citado por Ovejero, 1990) hablaba hace casi un siglo de las grandes ventajas que tiene la interacción entre compañeros para el desarrollo cognitivo a causa principalmente de los conflictos sociocognitivos a que lleva tal interacción, ya que "la formación de las operaciones necesita siempre un entorno favorable a la "cooperación", es decir, a las operaciones realizadas en común (por ejemplo, el papel que juega la discusión, la crítica mutua, los problemas suscitados por el intercambio de información, la curiosidad aguzada por la influencia cultural de un grupo social, etc.)" (Piaget, citado por Ovejero, 1990, 68).

A partir de aquí, los autores de la escuela de Ginebra, entre los que se destacan Mugny y Doise, Pret-Clermont, Mugny y Pérez, proporcionaron la base y desarrollaron el concepto de la cooperación que había introducido Piaget, adoptando siempre un punto de vista pedagógico.

Célestin Freinet es también uno de los principales promotores de la cooperación en la escuela, constituyendo el eje básico de su pedagogía popular. Sin embargo, siguiendo a Ovejero $(1990,63)$, cabe remarcar que, como miembro de la Escuela Nueva y el movimiento de Renovación Pedagógica, la cooperación para Freinet surgió de las dudas en cuanto a la eficacia de las técnicas pedagógicas tradicionales y se basó fundamentalmente en la cooperación entre maestros o entre alumno y maestro, así como en la reducción de las clases magistrales (Ovejero, 1990,66).

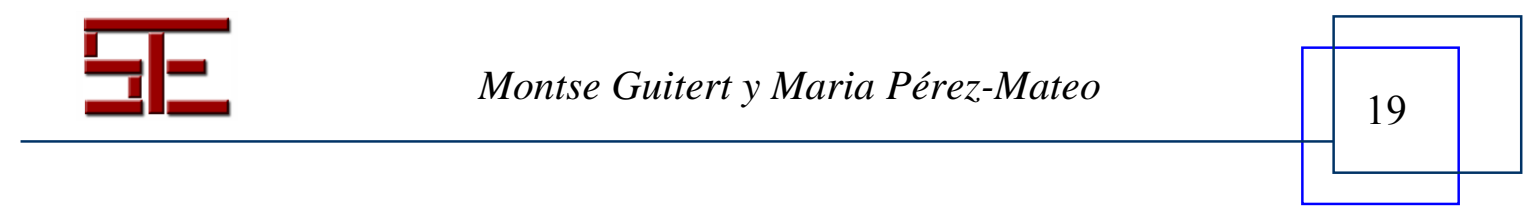




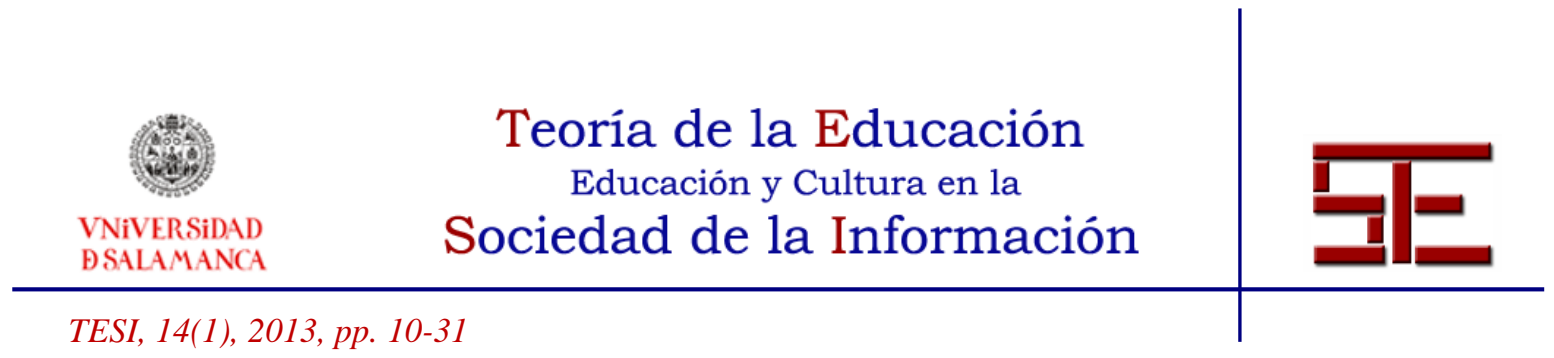

Aunque Piaget y Freinet destacan entre los representantes del movimiento cooperativo, son muchos los antecedentes que podríamos mencionar en el campo de la pedagogía.

En el ámbito internacional, cabe destacar a Rousseau, Neill, Makarenko y Cousinet y a Ferrer i Guàrdia y Rué en el ámbito nacional.

La Tabla 1 recoge algunos autores asociados al modelo cooperativo y la definición que realizan del término.

\begin{tabular}{|c|c|}
\hline AUTORES & DEFINICIÓN \\
\hline $\begin{array}{l}\text { Johnson y } \\
\text { Johnson } \\
(\mathbf{2 0 0 1 , 1 )}\end{array}$ & $\begin{array}{l}\text { Uso educativo de grupos reducidos en los cuales los estudiantes trabajan juntos para maximizar su } \\
\text { propio aprendizaje y el de los otros. }\end{array}$ \\
\hline $\begin{array}{l}\text { Sharan } \\
(1994,336)\end{array}$ & nfoque centrado en los grupos y el estudiante para la enseñanza y el aprendizaje en el aula. \\
\hline $\begin{array}{l}\text { Slavin }(1987, \\
\text { 116) }\end{array}$ & $\begin{array}{l}\text { Conjunto de métodos de instrucción en los cuales se motiva o requiere a los estudiantes trabajar } \\
\text { juntos en tareas académicas. }\end{array}$ \\
\hline $\begin{array}{l}\text { Deutsch } \\
(1949)\end{array}$ & $\begin{array}{l}\text { Aquella situación en la que las metas de los individuos separados van tan unidas que existe una } \\
\text { correlación positiva entre las consecuciones o logros de sus objetivos, de tal forma que un individuo } \\
\text { alcanza su objetivo si y sólo si también los otros participantes alcanzan el suyo. }\end{array}$ \\
\hline $\begin{array}{l}\text { Rué (1998, } \\
\text { 20) }\end{array}$ & $\begin{array}{l}\text { Producción en común en la cual cada componente ha desarrollado un rol específico, unas } \\
\text { habilidades, ha seguido un proceso, a la vez diferenciado y complementario, y tan necesario como } \\
\text { los del resto de componentes del pequeño grupo. La interpretación de una pieza musical por parte } \\
\text { de un trío es una buena imagen para ejemplificar un trabajo cooperativo. }\end{array}$ \\
\hline $\begin{array}{l}\text { Guitert } \quad y \\
\text { Giménez } \\
(\mathbf{2 0 0 0 , 1 1 4 )}\end{array}$ & $\begin{array}{l}\text { Se lleva a cabo un aprendizaje cooperativo cuando se da una reciprocidad entre un conjunto de } \\
\text { individuos que saben diferenciar y contrastar sus puntos de vista de tal manera que llegan a generar } \\
\text { un proceso de construcción de conocimiento. Es un proceso en el que cada individuo aprende más } \\
\text { de lo que aprendería por sí solo, fruto de la interacción de los integrantes del equipo. }\end{array}$ \\
\hline $\begin{array}{l}\text { Suárez } \\
\text { (2004) }\end{array}$ & $\begin{array}{l}\text { Es una estrategia pedagógica que busca estructurar y fomentar la intersubjetividad, a través de la } \\
\text { interacción recíproca entre alumnos conformados en equipos, como condición social de } \\
\text { aprendizaje, de tal forma que al trabajar juntos todos y cada uno de sus integrantes puedan avanzar } \\
\text { a niveles superiores en su desarrollo. }\end{array}$ \\
\hline
\end{tabular}

Tabla 1. Definiciones de aprendizaje cooperativo

\section{3.- Origen y concepto de colaboración}

Al contrario que la cooperación, el modelo colaborativo encuentra sus raíces en la tradición educativa europea, concretamente en la anglosajona.

Siguiendo a Ovejero (1990), si Piaget y sobre todo los autores de la Escuela de Ginebra subrayaban la construcción social de la inteligencia y, por tanto, la importancia de la

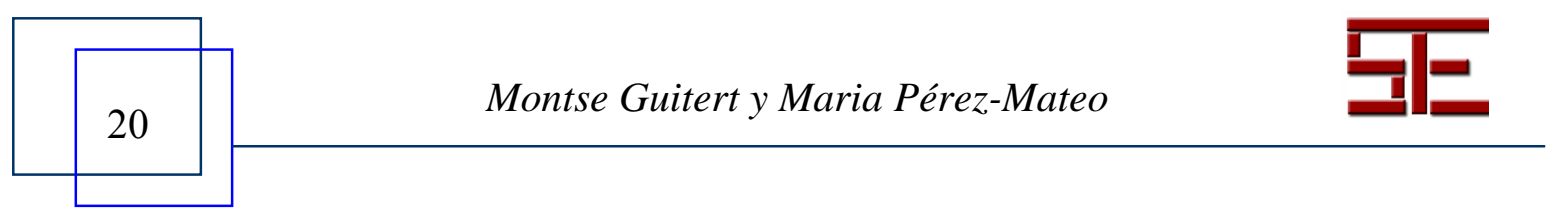




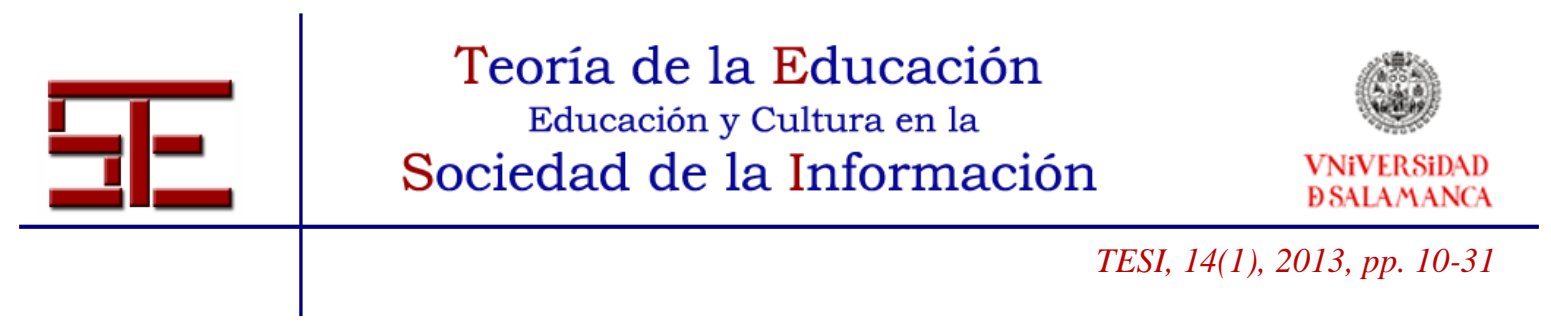

interacción social y la actividad común cooperativa para el mayor y mejor desarrollo intelectual, también Vygotsky propuso algo similar, dando gran importancia a los evidentes fundamentos sociales que posee el conocimiento.

Sin embargo y enfocado desde una vertiente psicológica, Vygotsky adoptó el término "colaboración", llegando incluso a mantener una postura opuesta a la de Piaget, puesto que para Vygotsky (Ovejero, 1990) (defensor de la teoría socio-cultural) la importancia reside más en la estrecha conexión que se postula entre el desarrollo intelectual y cognitivo, por una parte, y la interacción social por la otra más que en el desarrollo del razonamiento lógico y la adquisición de contenidos escolares, gracias a un proceso de reorganización cognitiva provocado por el surgimiento de conflictos y por su superación que propugnaba Piaget (defensor de la teoría socio-constructivista).

A partir de esta idea fundamental desarrollada por Vygotsky, diferentes autores defensores de este modelo han desarrollado una gran diversidad de definiciones, características e implicaciones en el ámbito educativo. Autores o estudiosos del modelo colaborativo son, en el terreno internacional, Dillenbourg, Dimitriadis, Lewis, Brufee, Crook, Harasim, Hiltz y Turoff y, en el ámbito nacional, Gros, Salinas y Rubia.

La Tabla 2 recoge algunos autores asociados al modelo colaborativo y la definición que realizan del término.

\begin{tabular}{|c|c|}
\hline AUTORES & DEFINICIÓN \\
\hline $\begin{array}{l}\text { Dillenbourg } \\
\text { (1999) }\end{array}$ & Situación en la cual dos o más personas aprenden o intentan aprender algo juntos. \\
\hline $\begin{array}{l}\text { Bruffee } \\
(1993,3)\end{array}$ & $\begin{array}{l}\text { Un proceso reculturativo que ayuda a los estudiantes a convertirse en miembros de comunidades de } \\
\text { conocimiento cuya propiedad común es diferente de la propiedad común de las comunidades de } \\
\text { conocimiento a las que ya pertenecen. }\end{array}$ \\
\hline $\begin{array}{l}\text { Roschelle y } \\
\text { Teasley } \\
(\mathbf{1 9 9 5 , 7 0 )}\end{array}$ & $\begin{array}{l}\text { Una actividad coordinada y sincrónica, que surge como resultado de un intento continuo por } \\
\text { construir y mantener una concepción compartida de un problema. }\end{array}$ \\
\hline $\begin{array}{l}\text { Harasim et } \\
\text { al. } \quad(\mathbf{2 0 0 0}, \\
\text { 51) }\end{array}$ & $\begin{array}{l}\text { Cualquier actividad en la cual dos o más personas trabajan de forma conjunta para definir un } \\
\text { significado, explorar un tema o mejorar competencias. }\end{array}$ \\
\hline $\begin{array}{l}\text { Koschmann } \\
\text { (1996) }\end{array}$ & Una situación en la cual los estudiantes se involucran en resolver problemas juntos. \\
\hline $\begin{array}{l}\text { Driscoll y } \\
\text { Vergara } \\
(1997,91)\end{array}$ & $\begin{array}{l}\text { Para que exista un verdadero aprendizaje colaborativo, no sólo se requiere trabajar juntos, sino } \\
\text { cooperar en el logro de una meta que no se puede lograr individualmente. }\end{array}$ \\
\hline $\begin{array}{l}\text { Gros } \quad y \\
\text { Adrián } \\
(\mathbf{2 0 0 4 )}\end{array}$ & $\begin{array}{l}\text { Proceso de constante interacción en la resolución de problemas, elaboración de proyectos o en } \\
\text { discusiones acerca de un tema en concreto; donde cada participante tiene definido su rol de }\end{array}$ \\
\hline
\end{tabular}

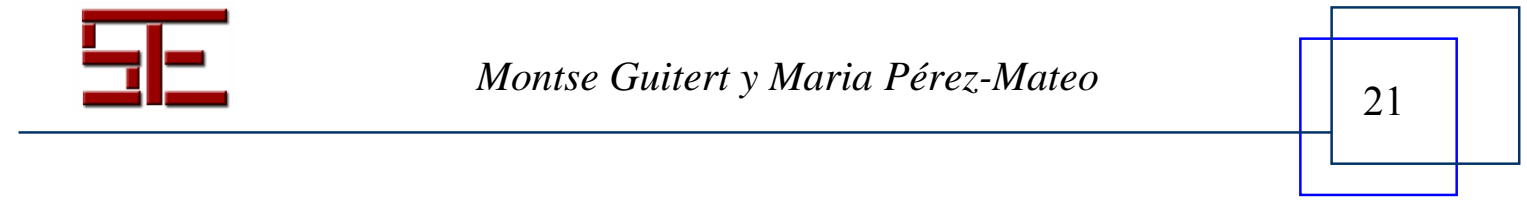




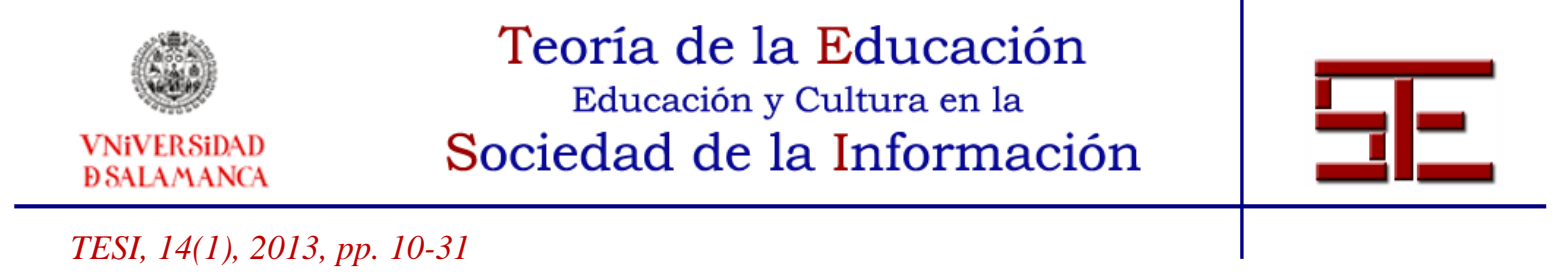

\begin{tabular}{|l|l|}
\hline & $\begin{array}{l}\text { colaborador en el logro de aprendizajes compartidos, y donde el profesor igualmente participa como } \\
\text { orientador y mediador, garantizando la efectividad de la actividad colaborativa. }\end{array}$ \\
\hline $\begin{array}{l}\text { Salinas } \\
(\mathbf{2 0 0 0 , 2 0 0 )}\end{array}$ & Adquisición de destrezas y actitudes que ocurren como resultado de la interacción en grupo. \\
\hline
\end{tabular}

Tabla 2. Definiciones de aprendizaje colaborativo

\section{4.- Similitudes entre cooperar o colaborar}

A pesar de los estudios que analizan las diferencias entre ambos modelos, es frecuente observar en la literatura del ámbito un uso indiferenciado de los términos "cooperar" y "colaborar". Se observan dos situaciones concretas en este aspecto.

Por un lado, algunos autores los utilizan como sinónimos, es decir, utilizan ambos conceptos sin apuntar diferencias entre ambos o especificidades. Por tanto, en algunas ocasiones se habla de cooperación y en otras de colaboración para referirse al mismo hecho.

Por otro lado, también es frecuente que los autores se decanten por uno de los conceptos y lo desarrollen a partir de elementos de ambos modelos. Por ejemplo, McConnell (2000, 8) señala que "aunque la distinción es a veces útil, utilizaré el término "aprendizaje cooperativo" en este libro para referirme a ambas formas de aprendizaje". Johnson, Johnson y Holubec (1999) identifican 5 elementos clave para que pueda desarrollarse un verdadero aprendizaje cooperativo; sin embargo, es frecuente en la literatura educativa (Driscoll y Vergara, 1997; Zañartu, 2003) su referencia para definir aprendizaje colaborativo.

\section{4.- HACIA UNA DEFINICIÓN DE APRENDIZAJE COLABORATIVO EN ENTORNOS VIRTUALES}

A la luz de los elementos expuestos anteriormente podemos concluir que cooperación y colaboración son dos corrientes paralelas con orígenes y tradiciones diferentes pero que, en determinados contextos o situaciones, son utilizados como sinónimos.

Cabe destacar en este punto el importante rol que ha ejercido la introducción de las TIC al ámbito educativo.

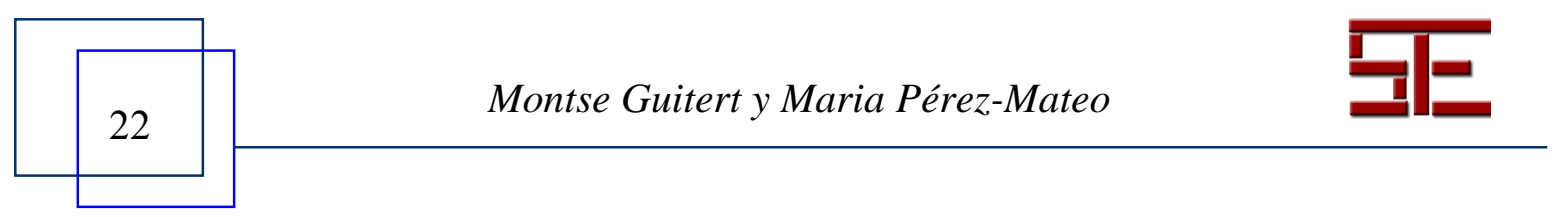




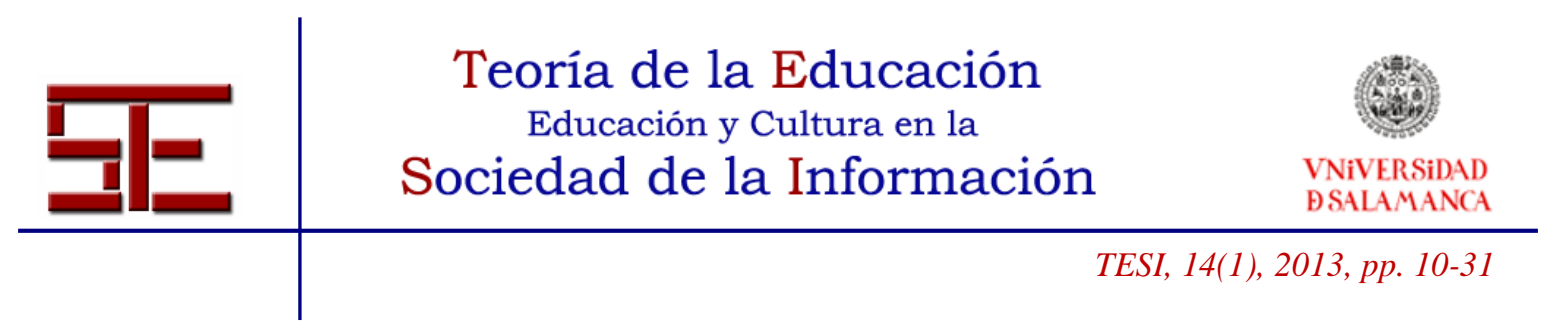

Los antecedentes en el ámbito, basados en la tradición pedagógica de trabajo en grupo, hacían referencia a la cooperación y el concepto educativo de trabajo entre iguales. Sin embargo, siguiendo a Ovejero $(1990,66)$, aunque entre los pedagogos existen muchos e importantes antecedentes del aprendizaje cooperativo, han sido sobre todo los psicólogos sociales los que han trabajado este tema [...] subrayando los fundamentos teóricos, psicológicos y principalmente psicosociales que explican la eficacia de ese aprendizaje, fundamentos que básicamente están en Piaget y mucho más aún en Vygotsky y G. H. Mead.

Sin embargo, a partir de la integración de las TIC a la educación, los numerosos estudios de talle psicológico han acabado por desplazar el término cooperativo y ensalzando la colaboración. Así pues, las aportaciones de tecnólogos educativos e informáticos parecen haber desplazado a la cooperación en pro de la colaboración.

Efectivamente, la revisión bibliográfica sobre el aprendizaje en línea (Romeu, 2011) y los procesos de aprendizaje conjunto entre estudiantes (Romero, 2008; Guitert et al., 2005; Pérez-Mateo, 2010) pone de manifiesto que la mayoría de estudios se refieren al término "colaboración", el cual se nutre, no obstante, de ambos modelos. Constatamos, por tanto, que, actualmente, existe una tendencia hacia el uso del término "colaboración", al cual se le atribuye el grado máximo de trabajo conjunto.

No obstante, lejos de elucidar respuestas satisfactorias para los expertos en educación sobre esta cuestión, el debate sobre qué es aprendizaje cooperativo, qué es aprendizaje colaborativo y cuáles son sus puntos de encuentro y diferencias está todavía abierto.

Es más, actualmente no quedan claras las características y limitaciones de cada modelo. Así, partidarios y seguidores de ambos modelos aseguran que es a través de aquél que se puede hablar de un trabajo conjunto de alta intensidad y reciprocidad.

Asistimos, pues, a un momento de confusión en los conceptos. Consideramos que en este proceso se han invertido los significados entre conceptos. Para algunos educadores, el trabajo conjunto intenso es la cooperación; para otros educadores y, en general, adeptos a entornos virtuales, esta intensidad se representa por la colaboración.

Estos elementos, en los cuales confluyen influencias de ambos modelos, sirven como base para la elaboración de una definición así como para ayudarnos a identificar qué nomenclatura utilizar en RACEV.

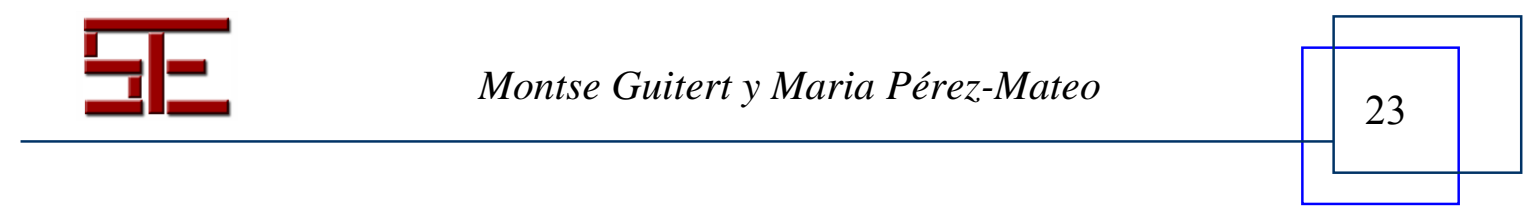




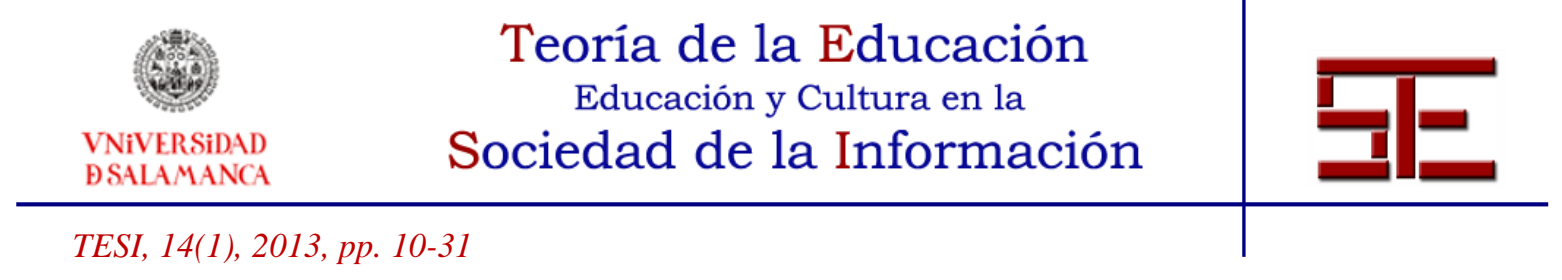

Teniendo en cuenta que a partir de la introducción de las TIC a la educación la colaboración ha ganado terreno frente a otros términos o modelos, desde la Red Temática sobre Aprendizaje Colaborativo Virtual adoptamos el término "colaborativo" para referirnos a esta metodología de aprendizaje. A este término colaborativo le atribuimos, sin embargo, diferentes grados o intensidades de colaboración (Figura 3) en función del grado de estructuración de la actividad (por parte del docente en función de las orientaciones y guía) y la intensidad de los procesos de intercambio (por parte del estudiante; la intensidad entre estudiantes define el grado de construcción conjunta de conocimiento).

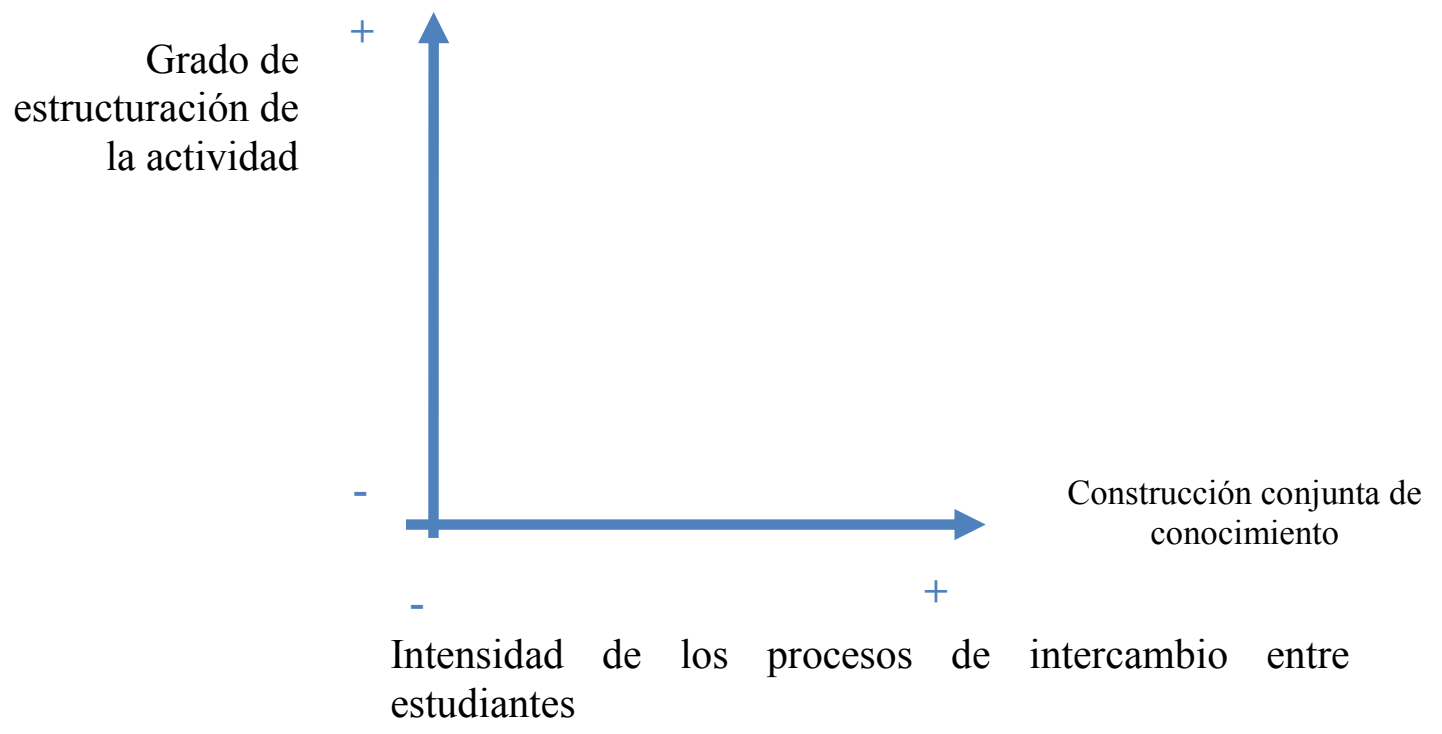

Figura 3. Grados de colaboración

La combinación de ambos elementos abarca diferentes formas y grados de colaboración. Por ejemplo, el trabajo por proyectos implica un alto grado de intercambio entre estudiantes y una estructuración media. Un debate conlleva un menor grado de estructuración y de construcción entre estudiantes.

Basándonos en las definiciones expuestas anteriormente, definimos la colaboración de alta intensidad como:

Un proceso compartido, coordinado e interdependiente, en el cual los estudiantes trabajan juntos para alcanzar un objetivo común en un entorno virtual. El aprendizaje colaborativo se basa en un proceso

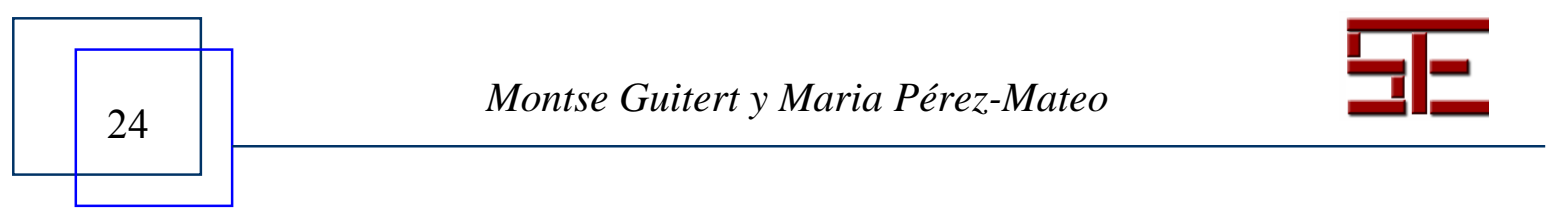




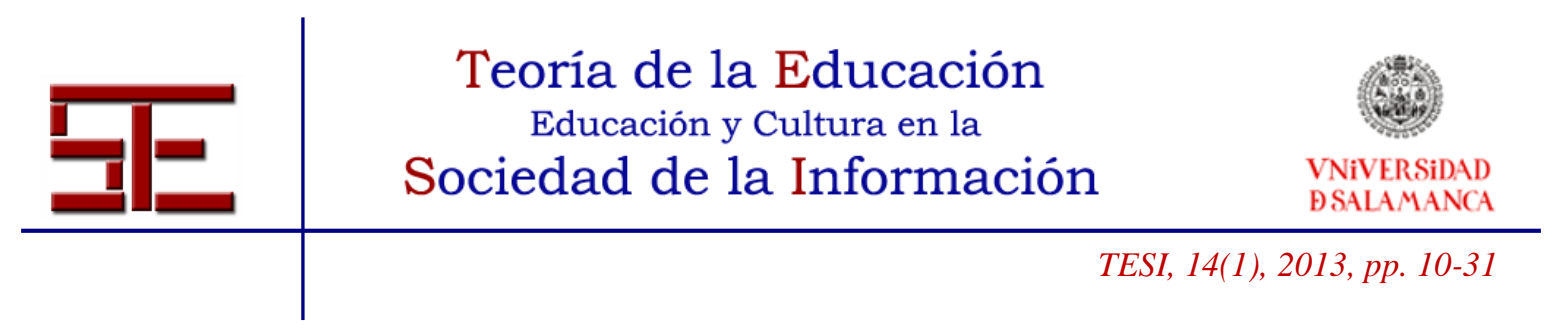

de actividad, interacción y reciprocidad entre los estudiantes, facilitando la construcción conjunta de significados y un avance individual hacia niveles superiores de desarrollo (RACEV).

Cuando el aprendizaje colaborativo tiene lugar en el entorno virtual, el estudiante dispone de un conjunto de herramientas tecnológicas que favorecen la consecución de este proceso. La condición de deslocalización geográfica y temporal implica al mismo tiempo la necesidad de reparar en instrumentos de gestión del proceso de colaboración que tienden a ser omitidos en un contexto presencial como son normativas de procedimiento o una planificación detallada del trabajo a realizar y que favorecen y optimizan tanto su desarrollo como los resultados (Guitert et al., 2005; Pérez-Mateo, 2010). De acuerdo con Román (2003) el trabajo colaborativo en entornos virtuales es una labor cualitativamente mejor que el planteado por los esquemas de organización tradicionales.

De esta manera, cuando el concepto de aprendizaje colaborativo se extiende en el entorno virtual, el concepto permanece pero las condiciones, y por ello las posibilidades, cambian sustancialmente. El entorno virtual inaugura oportunidades innovadoras para la colaboración, la comunicación y la producción de conocimientos y aumenta las posibilidades para poder aprender y trabajar en equipo a las cuales se veía limitada hasta ahora en un entorno de trabajo presencial (Harasim et al., 2000).

Es por ello que ya sea refiriéndonos a la cooperación o a la colaboración en educación, no se cuestiona que el aprendizaje y en general cualquier actividad desarrollada en un mundo global no puede entenderse si no es a partir de la interacción con otros estudiantes.

\section{5.- BIBLIOGRAFÍA}

Area, M. (2006). Autoformación del profesorado. Colaboración a través de la red. Práctica docente, 3.

Badia, A. \& García, C. (2006). Incorporación de las TIC en la enseñanza y el aprendizaje basados en la elaboración colaborativa de proyectos. Revista de Universidad y Sociedad del Conocimiento, 3 (2), 42-54.

Brindley, J. E., Walti, C. \& Blaschke, L. M. (2009). Creating effective collaborative learning groups in an online environment. International Review of Research in Open and Distance Learning, 10 (3), 1-18.

Bruffee, K. (1993). Collaborative learning. Baltimore: Johns Hopkins University Press.

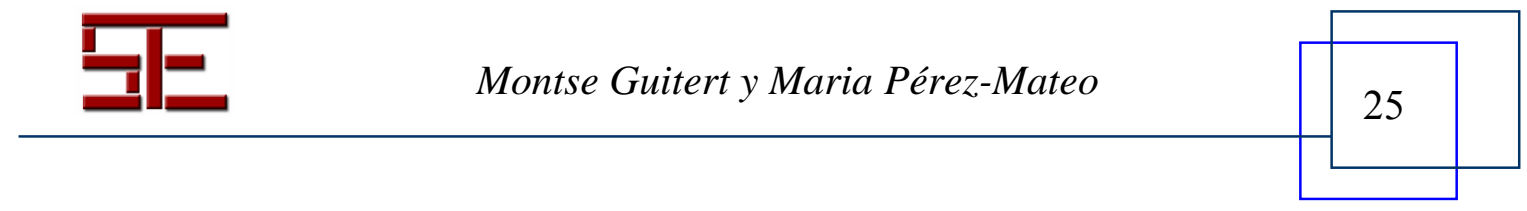




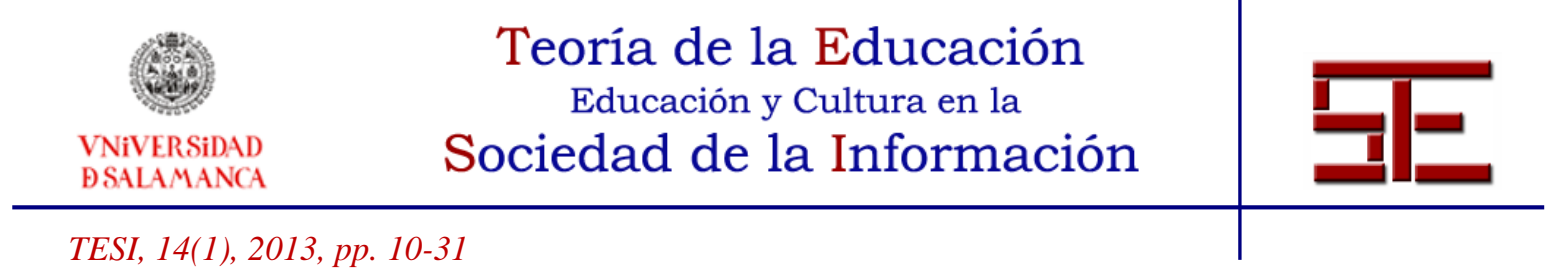

Brush, T. A. (1998). Embedding cooperative learning into the design of integrated learning systems: Rationale and guidelines. Educational Technology Research and Development, 46 (3), 5-18.

Crook, C. (1998). Ordenadores y aprendizaje colaborativo. Madrid: Morata.

Curtis, D. D. \& Lawson, M. J. (2001). Exploring collaborative online learning. Journal of Asynchronous Learning Networks, 5 (1), 21-34.

Dalsgaard, C. \& Paulsen, M. F. (2009). Transparency in cooperative online education. International Review of Research in Open and Distance Learning, 10 (3).

Deutsch, M. (1949). A theory of cooperation and competition. Human Relations, 2, 129152.

Dillenbourg, P. (1999). Collaborative learning: Cognitive and computational approaches. New York: Elsevier Science.

(2002). Over-scripting CSCL: The risks of blending collaborative learning with instructional design. En P. A. Kirschner (Ed.), Inaugural address, three worlds of CSCL. Can we support CSCL? (pp. 61-91). Heerlen: Open Universiteit Nederland.

(2003). Preface. En J. Andriessen, M. Baker y D. Suthers (Eds.), Arguing to learn: Confronting cognitions in computer-supported collaborative learning environments (pp. vii-ix). Kluwer: Dordrecht.

Driscoll, M. P. \& Vergara, A. (1997). Nuevas Tecnologías y su impacto en la educación del futuro. Pensamiento Educativo, 21.

Garrison, D. R. (2006). Online collaboration principles. Journal of Asynchronous Learning Networks, 10 (1), 25-34.

Garrison, D. R., Anderson, T. \& Archer, W. (2000). Critical inquiry in a text-based environment: Computer conferencing in higher education. The Internet and Higher Education, 2, 87-105.

González, J. \& Wagenaar, R. (2003). TUNING. Educational Structures in Europe. Informe final. Extraído el 1 de febrero, 2012, de

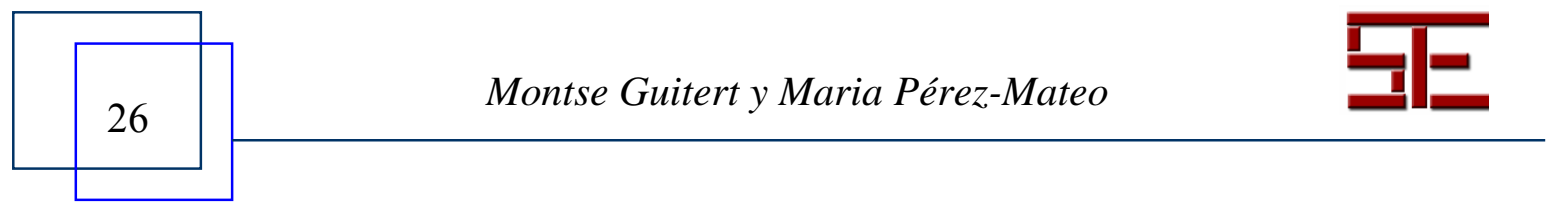




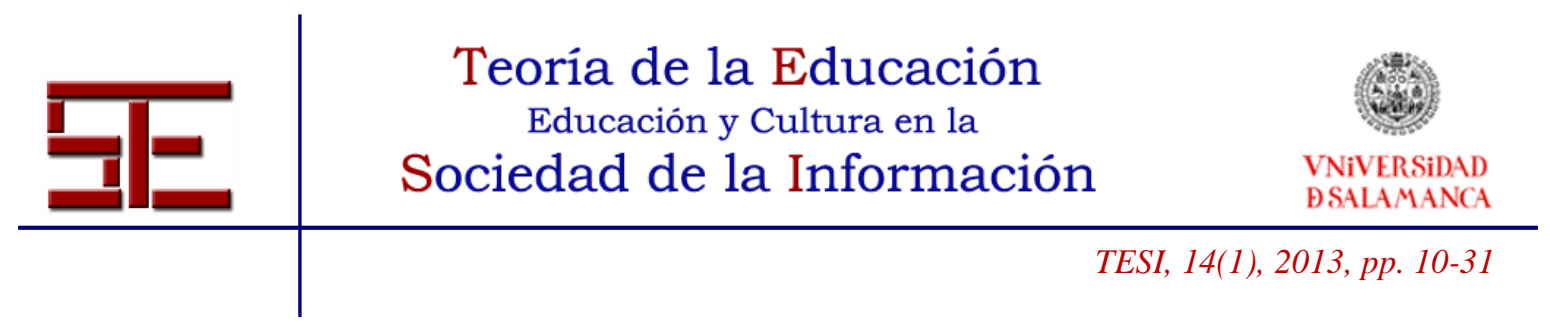

http://www.relint.deusto.es/TUNINGProject/spanish/doc_fase1/Tuning\%20Educational .pdf .

Gros, B. \& Adrián, M. (2004). Estudio sobre el uso de los foros virtuales para favorecer las actividades colaborativas en la enseñanza superior. Teoría de la Educación, 5.

Guitert, M. \& Giménez, F. (2000). El trabajo cooperativo en entornos virtuales de aprendizaje. En J. M. Duart y A. Sangrà (Eds.), Aprender en la virtualidad (pp. 113-134). Barcelona: Gedisa.

Guitert, M., Lloret, T., Giménez, F. \& Romeu, T. (2005). El treball i l'aprenentatge cooperatius en entorns virtuals: El cas de la Universitat Oberta de Catalunya (UOC). Coneixement i Societat. Revista d'Universitats, Recerca i Societat De La Informació, 8, 44-77.

Guitert, M., Romeu, T. \& Pérez-Mateo, M. (2007). Competencias TIC y trabajo en equipo en entornos virtuales. Revista de Universidad y Sociedad del Conocimiento, 4 (1).

Gunawardena, C. N. \& Zittle, F. (1997). Social presence as a predictor of satisfaction within a computer-mediated conferencing environment. American Journal of Distance Education, 11, 8-26.

Harasim, L., Hiltz, S. R., Turoff, M. \& Teles, L. (2000). Redes de aprendizaje. Guía para la enseñanza y el aprendizaje en red. Barcelona: Gedisa.

Haythornthwaite, C. (2006). Facilitating collaboration in online learning. Journal of Asynchronous Learning Networks, 10 (1), 7-23.

Holliman, R. \& Scanlon, E. (2006). Investigating cooperation and collaboration in near synchronous computer mediated conferences. Computers \& Education, 46 (3), 322-335.

Johnson, D. W. \& Johnson, R. T. (1989). Cooperation and competition: Theory and research. Edina, MN: Interaction Book Company.

(1994). Joining together: Group theory and group skills. Boston: Allyn y Bacon.

(2001). The Cooperative Learning Institute.

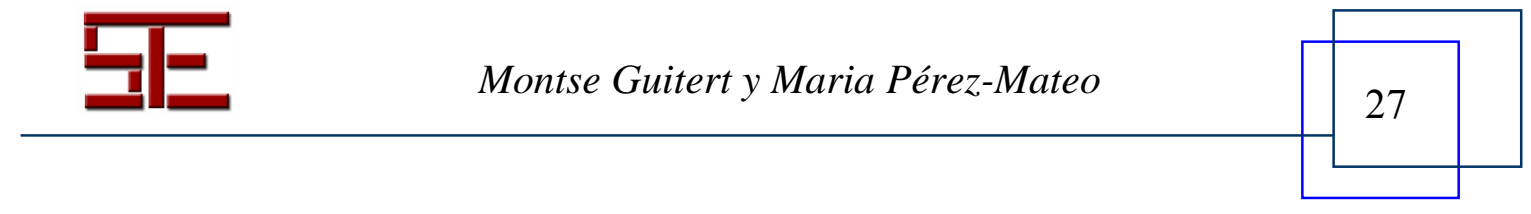




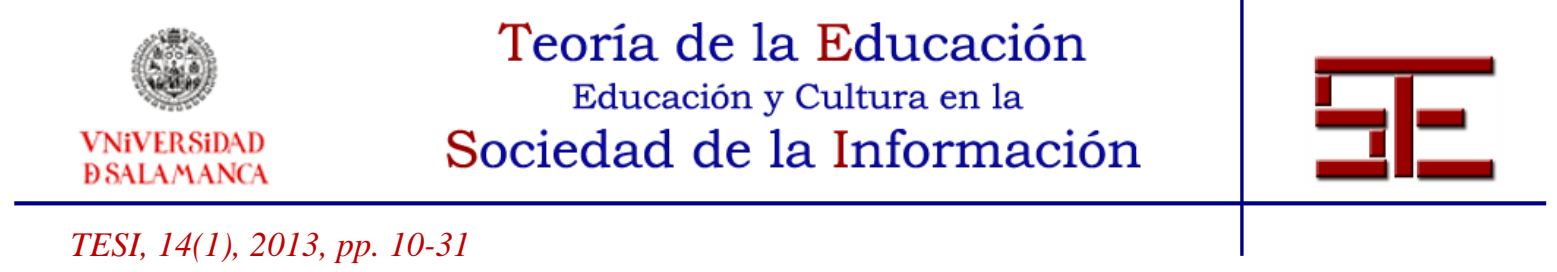

Johnson, D., Johnson, R. \& Holubec, E. (1999). El aprendizaje cooperativo en el aula. Barcelona: Paidós.

Jonassen, D. (1994). Toward a constructivist design model. Educational Technology, 34 (4), 34-37.

Kirschner, P. A. (2002). Three worlds of CSCL. Can we support CSCL. Heerlen: Open University of the Netherlands.

Koschmann, T. (1996). Paradigm shifts and instructional technology. En T. Koschmann (Ed.), CSCL: Theory and practice of an emergin paradigm (pp. 1-23). Mahwah, New Jersey: Lawrence Erlbaum.

Kreijns, K., Kirschner, P. A. \& Jochems, W. (2003). Identifying the pitfalls for social interaction in computer-supported collaborative learning environments: A review of the research. Computers in Human Behavior, 19, 335-353.

Lara, S. (2001). Una estrategia eficaz para fomentar la cooperación. Estudios sobre Educación, 1, 99-110.

Majó, J. \& Marquès, P. (2002). La revolución educativa en la era internet. Barcelona: CissPraxis.

McConnell, D. (2000). Implementing computer supported cooperative learning. London: Kogan Page.

(2006). E-learning groups and communities. Poland: The Society for Research into Higher Education y Open University Press.

Molinari, D. L. (2004). The role of social comments in problem-solving groups in an online class. American Journal of Distance Education, 18, 89-101.

Ovejero, A. (1990). El aprendizaje cooperativo. una alternativa eficaz a la enseñanza tradicional. Barcelona: PPU.

Panitz, T. (1997). Collaborative versus cooperative learning - a comparison of the two concepts which will help us understand the underlying nature of interactive learning. Cooperative Learning and College Teaching, 8 (2).

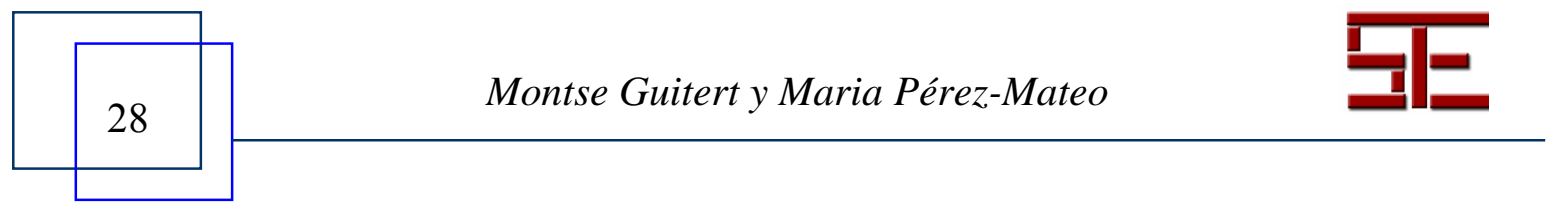




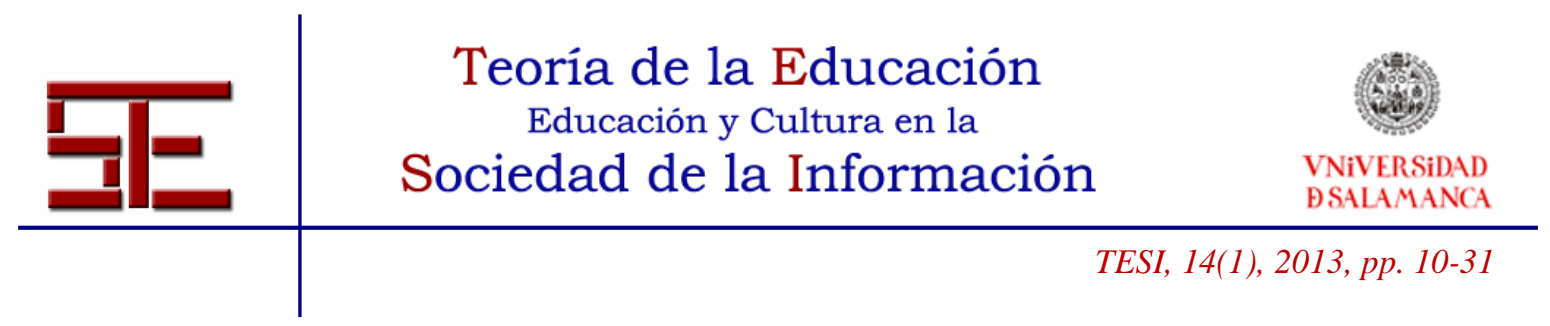

Pérez-Mateo, M. (2010). La dimensión social en el proceso de aprendizaje colaborativo virtual: el caso de la UOC [en línea]. Tesis doctoral. Extraído el 1 de febrero, 2012, de http://www.tdx.cat/handle/10803/37113.

Roberts, T. S. (2005). Computer-supported collaborative learning in higher education: An introduction. En T. S. Roberts (Ed.), Computer-supported collaborative learning in higher education (pp.1-18). Hershey: Idean Group Publishing.

Román, P. (2003). Posibilidades formativas de las herramientas groupware. El aprendizaje colaborativo en la educación. II Congreso Internacional Internet en la Educación.

Romero, M. (2008). Disseny i avaluació d'un centre virtual de recursos de tecnologia educativa com a eina de formació dels mestres en l'ús de les TIC. Tesis doctoral no publicada. Universitat Rovira i Virgili (Tarragona).

Romeu, T. (2011). La docencia en colaboración en contextos virtuales. Estudio de caso de un equipo de docentes del área de competencias digitales de la UOC [en línea]. Tesis doctoral. Extraído el 1 de febrero, 2012, de http://www.tdx.cat/handle/10803/96768

Roschelle, J. \& Teasley, S. D. (1995). The construction of shared knowledge in collaborative problem solving. En: C. E. O'Malley (Ed.), Computer-supported collaborative learning (pp. 69-197). Berlin: Springer-Verlag.

Rué, J. (1998). El aula: Un espacio para la cooperación. En C. Mir (Ed.), Cooperar en la escuela: La responsabilidad de educar para la democracia (pp. 17-50). Barcelona: Graó.

Salinas, J. (2000). El aprendizaje colaborativo con los nuevos canales de comunicación. En J. Cabero (Ed.), Nuevas tecnologías aplicadas a la educación (pp. 199-227). Madrid: Síntesis.

Sharan, S. (1994). Cooperative learning and the teacher. En S. Sharan (Ed.), Handbook of cooperative learning methods (pp. 336-348). Wesport: Greenwood Press.

Slavin, R. E. (1987). Cooperative learning: Where behavioral and humanistic approaches to classroom motivation meet. Elementary School Journal, 88, 29-35.

Slavin, E. R., Rogers, C. \& Kutnick, P. (1992). Aprendizaje cooperativo. Psicología social de la escuela primaria. Barcelona: Paidós.

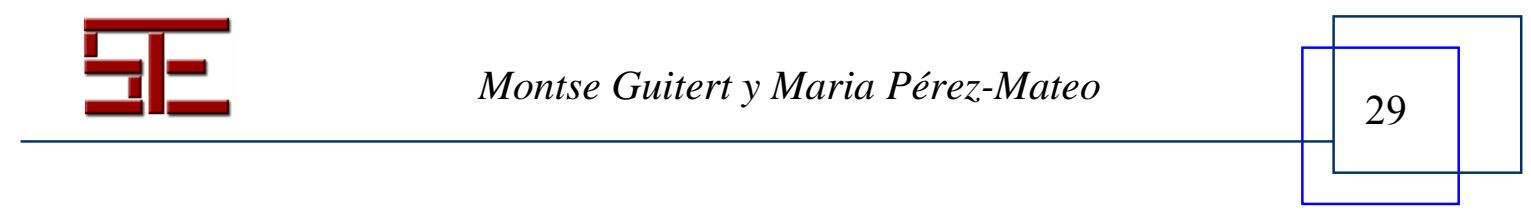




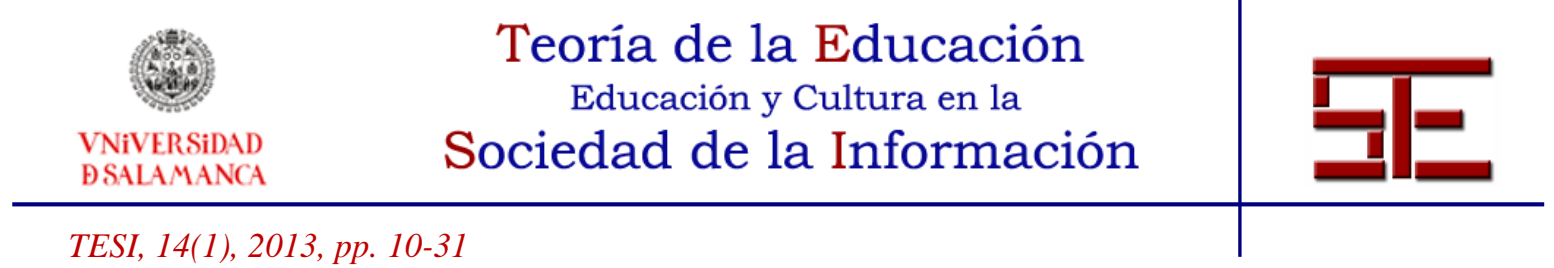

Suárez, C. (2004). La interacción cooperativa: Condición social de aprendizaje. Revista Educación, XII (23), 79-100.

(2009). Estructura didáctica virtual para Moodle. Revista DIM: Didáctica, Innovación y Multimedia, 13.

Swan, K. (2006). Online collaboration: Introduction to the special issue. Journal of Asynchronous Learning Networks, 10, 3-5.

Thompson, J. C. (2005). Cooperative learning in computer-supported classes. Tesis doctoral no publicada. University of Melbourne.

$\mathrm{Tu}, \mathrm{C} . \mathrm{H}$. (2004). Online collaborative learning communities: Twenty-one designs to building an online collaborative learning community. Westport, CT: Libraries Unlimited.

Wegerif, R. (1998). The social dimension of asynchronous learning networks. Journal of Asynchronous Learning Networks, 2(1), 34-49.

Zañartu, L. M. (2003). Aprendizaje colaborativo: Una nueva forma de diálogo interpersonal y en red. Contexto Educativo. Revista Digital De Educación y Nuevas Tecnologías, 28. Extraído el 1 de febrero, 2012, de http://www.quadernsdigitals.net/index.php?accionMenu=hemeroteca. VisualizaArticulo IU.visualiza\&articulo_id=278.

\section{Notas}

${ }^{1}$ Entre otros, Area (2006); Brindley, Walti, y Blaschke (2009); Crook (1998); Curtis y Lawson (2001); Garrison, Anderson, y Archer (2001); Guitert, Romeu, y Pérez-Mateo (2007); Harasim et al. (2000); Haythornthwaite (2006); Johnson y Johnson (1994); Jonassen (1994); Molinari (2004); Slavin, Rogers, y Kutnick (1992); Swan (2006); Tu (2004); Wegerif (1998).

${ }^{2}$ Con el agradecimiento al Ministerio de Ciencia e Innovación por la subvención recibida.

${ }^{3}$ En catalán, Universitat Oberta de Catalunya.

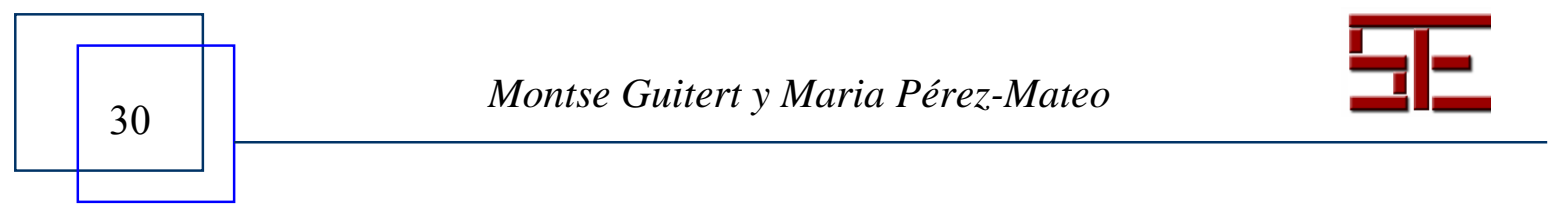




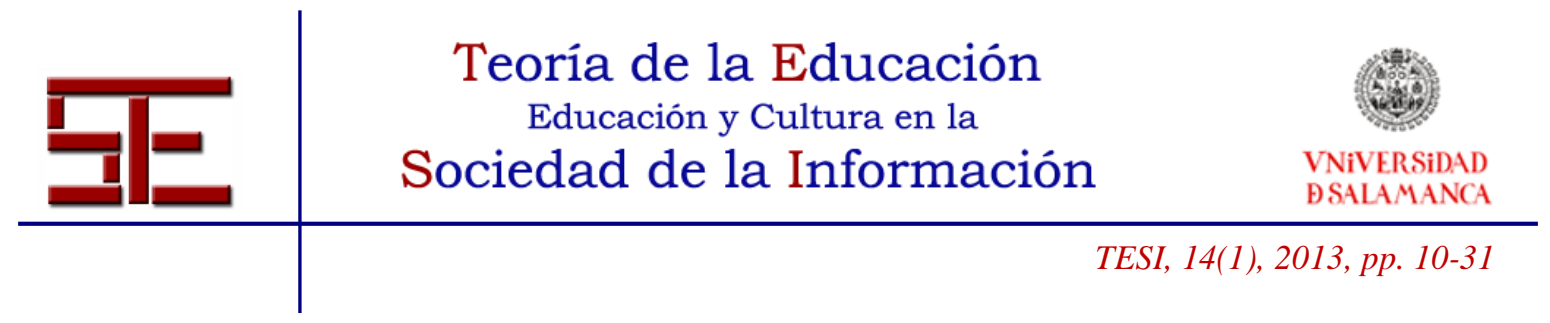

Para citar el presente artículo puede utilizar la siguiente referencia:

Guitert, M. y Pérez-Mateo, M. (2013). La colaboración en la red: hacia una definición de aprendizaje colaborativo en entornos virtuales. Revista Teoría de la Educación: Educación y Cultura en la Sociedad de la Información. 14(1), 10-30 [Fecha de consulta: $\mathrm{dd} / \mathrm{mm} /$ aaaa $]$.

http://campus.usal.es/ revistas_trabajo/index.php/revistatesi/article/view/9440/9730

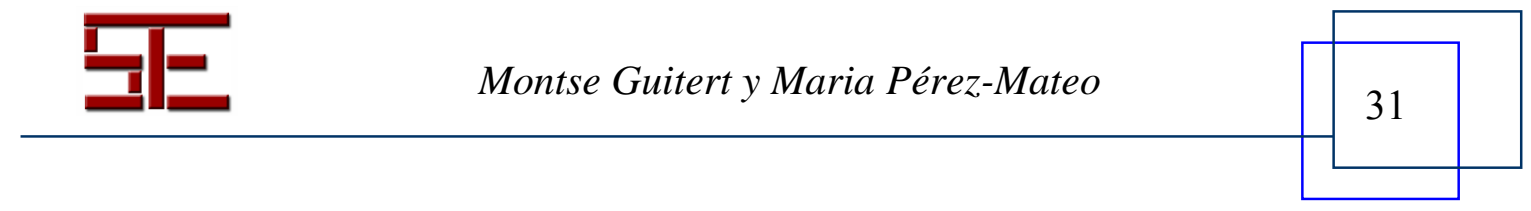

